\title{
Comparison of Prostate Cancer Detection Rates of Cognitive Fusion-targeted Biopsy and Standard Transrectal Ultrasound-guided Biopsy
}

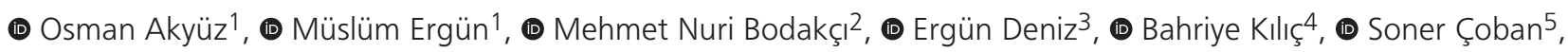 \\ ๑ Süleyman Sami Çakır ${ }^{1}$, (1) Ahmet Hamdi Tefekli ${ }^{1}$ \\ 1 Atlas University Medicine Hospital, Clinic of Urology, Istanbul, Turkey \\ ${ }^{2}$ Avcllar Hospital, Clinic of Urology, Istanbul, Turkey \\ ${ }^{3}$ Atlas University Medicine Hospital, Clinic of Radiology, Istanbul, Turkey \\ ${ }^{4}$ Atlas University Medicine Hospital, Clinic of Anaesthesiology and Reanimation, Istanbul, Turkey \\ 5 University of Health Sciences Turkey, Bursa Yüksek Ihtisas Training and Research Hospital, Clinic of Urology, Bursa, Turkey
}

\begin{abstract}
Objective: The aim of this study is to investigate whether there is a difference between standard prostate needle biopsies and cognitive fusion prostate biopsies (classical 12-core plus 2 cognitive lesions) for any prostate cancer or clinically significant prostate cancer (CSPCa) detection rates.

Materials and Methods: The records of 340 patients who underwent standard transrectal ultrasound-guided biopsy (TRUS-SB) and cognitive targeted biopsy (COG-TB) between June 2015 and January 2020 at our institute were retrospectively reviewed and included in the study. There were 185 patients in the TRUS-SB (12-core) group and 155 patients in the COG-TB (12-core +2 lesions) group. Patients undergoing COG-TB underwent 3-T multiparametric magnetic resonance imaging before the biopsy procedure and were evaluated with the prostate imaging reporting and data system version 2 . The rates of any prostate cancer and CSPCa were compared between groups.

Results: The patient mean ages were determined as $64.02 \pm 5.15$ and $64.41 \pm 3.93$ years for the TRUS-SB and COG-TABLO groups, respectively. Any prostate cancer was detected in $38 / 185$ patients (20.54\%) in the TRUS-SB group, and $44 / 155$ patients (28.38\%) in the COG-TB group. CSPCa rates were determined as $57.80 \%$ (22/38 patients), and 56.8\% (25/44) for the TRUS-SB and COG-TB groups, respectively. There was no statistically significant difference between the groups regarding cancer $(p=0.092)$ and CSPCa detection rates $(p=0.843)$.

Conclusion: In our study, no significant difference was found between the overall prostate cancer and clinically important prostate cancer detection rates between the TRUS-SB and COG-TB groups.

Keywords: Biopsy, cognitive fusion, standard biopsy prostate cancer, multiparametric magnetic resonance imaging
\end{abstract}

\section{Introduction}

Prostate cancer is the second most common cancer in men and ranks fifth among the causes of cancer-related deaths. It is reported that approximately 1.3 million patients worldwide have been diagnosed with prostate cancer in 2018 (1). The incidence of prostate cancer has started to increase, especially with the introduction of the prostate-specific antigen (PSA) test introduced in the 1980s. Transrectal ultrasound-guided standard prostate biopsy (TRUS-SB) is the gold standard method to diagnose cancer in patients with suspected prostate cancer suggested by increased PSA levels or detection of the prostatic nodule(s) during a digital rectal examination (DRE). Initially, a six-core systematic, sextant biopsy protocol was used (2). However, the number of cores obtained increased over time, with 12-14 cores being taken as standard practise (3). While cancer detection rates were reported to range between $27 \%$ and $44 \%$ with TRUS-SB, $15 \%$ to $34 \%$ of cancer cases may be overlooked (4). Although saturation biopsy procedures were started when 20 or more cores were obtained, only a limited increase in prostate cancer detection rates was achieved with this technique (5). TRUS-SB has the advantage of lower cost and faster application; however, it diagnoses an excessive number of clinically insignificant cancer (CISPCa) cases and leads to 
numerous unnecessary treatments. It also detects clinically significant cancers (CSPCa) at a lower rate and has limitations, such as false negativity (6). In parallel with the developments in magnetic resonance imaging (MRI), multiparametric prostate MRI (Mp-MRI), which provides information about the prostate's anatomical and functional structure has been started to be used to prevent these limitations. The aim is to increase clinically significant prostate cancer (CSPCa) detection rates by taking fewer samples from the prostate (7). MRI targeted fusion biopsies are performed in three ways:

1. Real-time MRI-guided biopsy [(MRI in bore- targeted biopsy (TB)]. 2. Fusion biopsy (MRI Ultrasound Fusion Software) (MRI/US-TB) combines MRI and ultrasound images using the software.

3. Cognitive biopsy (COG-TB) uses biopsy materials obtained from these suspicious areas after detecting suspicious lesions with Mp-MRI (8).

While performing a biopsy after MRI in all applications, there is also an application unity about adding 12 quadrant standard biopsies. Although there are studies in this direction, it is not recommended to take a biopsy only from the lesion (9). In this study, we aimed to evaluate whether there is a difference between standard TRUS-guided prostate needle biopsies and cognitive fusion prostate biopsies, regarding the detection rates of any prostate cancer and clinically significant prostate cancer.

\section{Materials and Methods}

Using our computerised database, the records of 377 patients who underwent TRUS-SB and COG-TB between June 2015 and January 2020 at our institute were retrospectively analysed. Patients with suspicious findings such as induration or nodules suggest prostate cancer on DRE or high PSA levels were included in the study. Patients with a large nodule detected during DRE suggesting suspected metastatic disease $(n=12)$ and patients with a previous history of negative biopsy $(n=25)$ were excluded from the study. A total of 340 patients, including 185 cases in the TRUS-SB group and 155 cases in the COG-TB group, were enrolled in the study. In the COG-TB group patients with suspected lesions detected on prebiopsy MP-MRI were also included in the study. MRI examinations were performed with three Tesla MRI units (Ge Healthcare 3T; Pioneer Signa MLG, Japan). T2-weighted, dynamic contrast diffusion-weighted images and visible diffusion coefficient maps were obtained. MRI images were examined by a single radiologist experienced in the field. Suspicious lesions were identified, and Prostate Imaging Reporting and Data System (PI-RADS) scoring was performed. (PI-RADS $1=$ Very low CSPCa is highly unlikely to be present, PI-RADS 2 =Low CSPCa is unlikely to be present, PI-RADS $3=$ Intermediate the presence of CSPCa is equivocal, PI-RADS $4=$ High CSPCa is likely to be present, PI-RADS $5=$ Very high CSPCa is highly likely to be present) (10). All biopsies were performed by the same urologist. Antibiotic prophylaxis with one gramme intravenous ceftriaxone and bowel prep were performed one hour before the procedure. After the patient was discharged, oral treatment with ciprofloxacin (500 mg twice a day) was maintained.

Biopsies were performed in the operating room by applying sedoanalgesia or caudal block. A 12-core systematic standard biopsy was performed in the TRUS-SB group by placing an endorectal TRUS probe (4-9 MHz endorectal probe PVT-781, Toshiba, Japan). Two more core biopsies were taken per suspected area in patients with suspicious findings on DRE or TRUS. In the COG-TB group, after inserting the TRUS probe, the suspicious lesions described on the Mp-MRI attempted to be identified. After taking two core biopsies per lesion from the regions with suspected areas, 12 systematic core biopsies were performed. An 18 Gauge $25 \mathrm{~cm}$ biopsy gun (Bard Monopty Biopsy gun) was used for biopsy. Tissues placed in $10 \%$ buffered formalin solution were sent to the laboratory for histopathological examination.

Any evidence of cancer, CSPCa and CISPCa rates were compared between the groups. There is still no consensus on the definition of CSPCa. For this reason, we preferred a frequently used definition when determining CSPCa rates. Based on the histopathology reports, prostate cancers with Gleason scores (GS) of $\geq 7$ were accepted as CSPCa. Similarly, GS 6, with a more than $5 \mathrm{~mm}$ tumour length in any of the cores, was considered CSPCa $(11,12)$.

\section{Statistical Analysis}

SPSS program (SPSS version 20.0; IBM, NY, USA) was used for statistical evaluation. Descriptive statistical methods (mean, standard deviation) and the chi-square test were used to evaluate the data. $\mathrm{P}<0.05$ was considered statistically significant. At all stages of the study, families were informed about the procedure, and informed consent forms were obtained. This study was conducted in accordance with the principles of the Helsinki Declaration and approval of the Ethics Committee of our institute was obtained (register no: 2018/15-14).

\section{Results}

The mean ages of patients included in the study were determined as $64.02 \pm 5.15$ and $64.41 \pm 3.93$ years for TRUS-SB and COG-TB groups, respectively. The mean serum PSA values were $11.83 \pm 4.13 \mathrm{ng} / \mathrm{mL}$ in the TRUS-SB group, and $9.1 \pm 5.37$ $\mathrm{ng} / \mathrm{mL}$ in the COG-TB group. The mean prostate volumes were $72.72 \pm 19.1$ and $71.51 \pm 24.3 \mathrm{~g}$ in the TRUS-SB and COGTB groups. The mean GS were determined as $6.40 \pm 0.8$ and $6.31 \pm 1.1$ for TRUS-SB and COG-TB groups. In the COG-TB group, the percentages of patients with PI-RADS 2, 3, 4 and 5 scores in Mp-MRI were $16.7 \%, 41.9 \%, 33.5 \%$ and $7.74 \%$, respectively. The lesion's mean length in the MRI $(\mathrm{mm})$ was determined as $14.27 \pm 3.1 \mathrm{~mm}$ (Table 1 ).

The average number of cores taken were determined as $2.90 \pm 0.4$ and 3.65 \pm 1.1 for TRUS-SB and COG-TB groups, respectively. The median positive cancer core length detected in the cores taken on biopsy was determined as $3.12 \pm 0.3 \mathrm{~mm}$ in TRUS-SB and $5.22 \pm 1.3 \mathrm{~mm}$ in COG-TB groups. Any prostate cancer was detected in 38/185 patients (20.54\%) in the TRUS-SB group and $44 / 155$ patients $(28.38 \%)$ in the COG-TB group. The CSPCa rates were determined as $57.80 \%$ (22/38 patients) and $56.8 \%$ (25/44) for TRUS-SB and COG-TB groups, respectively (Table 2). There was no statistically significant difference between the groups regarding cancer detection $(p=0.092)$ and CSPCa detection rates $(p=0.843)$. 


\begin{tabular}{|c|c|c|}
\hline & $\begin{array}{l}\text { TRUS-SB } \\
(n=185)\end{array}$ & $\begin{array}{l}\text { COG-TB } \\
(n=155)\end{array}$ \\
\hline Patient age (years) & $64.02 \pm 5.15$ & $64.41 \pm 3.93$ \\
\hline PSA value $(\mathrm{ng} / \mathrm{mL})$ & $11.83 \pm 4.13$ & $9.1 \pm 5.37$ \\
\hline Prostate volume (gramme) & $72.72 \pm 19.1$ & $71.51 \pm 24.3$ \\
\hline Mean Gleason score & $6.40 \pm 0.8$ & $6.31 \pm 1.1$ \\
\hline $\begin{array}{l}\text { Mean length of the lesion in the MRI } \\
(\mathrm{mm})\end{array}$ & - & $14.27 \pm 3.1$ \\
\hline PI-RADS 2 (n) & - & $26(16.7)$ \\
\hline PI-RADS 3 (n) & - & 65 (41.9) \\
\hline PI-RADS 4 (n) & - & $52(33.5)$ \\
\hline PI-RADS 5 (n) & - & $12(7.74)$ \\
\hline \multicolumn{3}{|c|}{$\begin{array}{l}\text { Data are presented as mean } \pm \text { standard deviation. } \\
\text { Data in parentheses represent percentages; n: Number of patients; mm: } \\
\text { Millimetre, TRUS-SB: Transrectal ultrasound-guided biopsy, COG-TB: Cognitive } \\
\text { targeted biopsy, PI-RADS: Prostate Imaging Reporting and Data System, MRI: } \\
\text { Magnetic resonance imaging }\end{array}$} \\
\hline
\end{tabular}

Table 2. Comparison of biopsy results and complications between the TRUS-SB and COG-TB groups

\begin{tabular}{|l|l|l|}
\hline & $\begin{array}{l}\text { TRUS-SB } \\
(\mathbf{n}=\mathbf{1 8 5})\end{array}$ & $\begin{array}{l}\text { COG-TB } \\
(\mathbf{n}=155)\end{array}$ \\
\hline No. cores (per patient) & $2.90 \pm 0.4$ & $3.65 \pm 1.1$ \\
\hline Median positive cancer core length (mm) & $3.12 \pm 0.3$ & $5.22 \pm 1.3$ \\
\hline Positive for any cancer (n) & $38(20.54 \%)$ & $44(28.38 \%)$ \\
\hline Positive for clinically significant cancer (n) & $22(57.80 \%)$ & $25(56.80 \%)$ \\
\hline Complications (n) & $30(16.21 \%)$ & $21(13.54 \%)$ \\
\hline Haematospermia & $12(6.48 \%)$ & $8(5.16 \%)$ \\
\hline -Urinary tract infections & $7(3.78 \%)$ & $5(3.22 \%)$ \\
\hline -Significant haematuria & $3(1.62 \%)$ & $3(1.93 \%)$ \\
\hline -Urinary retention & $5(2.70 \%)$ & $3(1.93 \%)$ \\
\hline -Sepsis & $2(1.08 \%)$ & $1(0.64 \%)$ \\
\hline -Significant rectal bleeding & $1(0.54 \%)$ & $1(0.64 \%)$ \\
\hline $\begin{array}{l}\text { Data in parentheses represent percentages, n: Number of patients, TRUS-SB: } \\
\text { Transrectal ultrasound-guided biopsy, COG-TB: Cognitive targeted biopsy }\end{array}$ \\
\hline
\end{tabular}

The complication rates were determined as $16.21 \%$ (30 patients) and $13.54 \%$ (21 patients) for TRUS-SB and COG-TB groups, respectively. The most common complication in the TRUS-SB group was haemospermia in 12 (6.48\%) patients. Infection is another common complication and was detected in seven (3.78\%) patients. Other complications were urinary retention in five $(2.70 \%)$, massive haematuria in three (1.62\%), urosepsis in two (1.08\%) patients, and massive rectal bleeding only in 1 $(0.54 \%)$ patient. Similarly, the most common complication in the COG-TB group was haemospermia in eight (5.16\%) patients. Infection was observed in five (3.22\%), urinary retention in three $(1.93 \%)$, massive haematuria in three $(1.93 \%)$, urosepsis in one $(0.64 \%)$ and massive recurrent rectal bleeding in only one $(0.64 \%)$ patient. In both groups, mild and moderate selflimiting haematuria and rectal bleeding were not considered complications and not included in the complication rates (Table
2). The mean operation time was determined as $9.2 \pm 0.8 \mathrm{~min}$ in the TRUS-SB and $11.3 \pm 1.8 \mathrm{~min}$ in the COG-TB groups.

\section{Discussion}

Prostate cancer is the second most common cancer in men (1) In the 1980s, the PSA was introduced, and the number of patients diagnosed started to increase. In a case of high PSA or the detection of prostatic induration on DRE, a biopsy is performed with suspicion of prostate cancer. Even asymptomatic patients undergo biopsy after the detection of increased PSA. Pathological examinations of patients who were operated for benign prostatic hyperplasia or the detection of undiagnosed cancer on autopsy have increased the prostate cancer incidence $(1,13)$. TRUS-SB was first introduced in 1989 by Hodge et al. (2) to assist in prostate cancer diagnosis. It is now used as the gold standard method in the diagnosis of prostate cancer. Although it has the advantage of quick application at low cost, it has disadvantages such as overestimating CISPCa and underestimating CSPCa. This leads to unnecessary and excessive treatment. These disadvantages were eliminated with the developments in MRI when the Mp-MRI started to be used. Suspected lesions regarding malignancy in the prostate were identified before the biopsy and started to be targeted during the procedure (14). The ideal prostate biopsy has been defined as being minimally invasive by taking fewer cores, having a low side effect profile, determining CSPCa in a high rate of patients, and minimising the CISCPCa detection rate to reduce unnecessary overtreatment (14).

In a recent systematic review and meta-analysis, Hu et al. (15) stated that MRI-guided biopsies are more useful than TRUS-Bx in detecting any cancer and CSPCa. They also reported that MRI-guided biopsies had a significantly higher detection rate in detecting any prostate cancer in patients with an initial biopsy. They reported that they did not detect a significant difference between the two groups regarding detecting any cancer and CSPC for patients with a previously negative biopsy (15). Schoots et al. (14) reported no significant difference in the rates of any cancer detection compared with TRUS-SB in biopsies performed with MRI guidance. However, the CSPCa detection rate was higher in the MRI group. In another study, Kasivisvanathan et al. (16) reported that in prostate cancer diagnosis that has not been previously biopsied, risk assessment with MRI, and then performing MRI-TB before the biopsy is more advantageous than TRUS-SB. Contrary to these studies, Baco et al. (17) reported no significant difference in the detection rate of CSPC between MRI-guided biopsies and TRUS-SB. In our study, no significant difference was found between COG-TB and TRUS-SB regarding detecting any prostate cancer and CSPCa in accordance with the results of Baco et al. (17). Although higher rates of any prostate cancer were detected in the COG-TB group, this was not significantly different from those detected in the TRUS-SB group ( $\mathrm{p}=0.092)$.

Although transrectal prostate needle biopsy is a safe procedure, it can sometimes cause serious complications, such as sepsis, which will require hospitalisation. Serious complications are rare and minor complications such as haematuria is more common (18). The most common complications after prostate biopsy 
are haematuria, haematospermia rectal bleeding, urinary tract infections and acute urinary retention (19). It has been reported that the rates of major complications such as massive haematuria, rectal bleeding or sepsis vary between $0.5 \%$ and $6.6 \%$, and the rates of complications requiring hospitalisation range between $0.5 \%$ and $4.8 \%(19,20)$. Our study's overall complication rates were $16.21 \%$ in the TRUS-SB group and $13.54 \%$ in the COG-TB group. Major complication rates were $3.24 \%$ and $3.22 \%$, respectively, in the TRUS-SB and COG-TB groups, consistent with the literature findings.

\section{Study Limitations}

The most important limitation of this study is its retrospective design. Prospective randomised studies comparing MRI COG and standard 12-core biopsy would help in this regard. However, only 12-core standard biopsies might not be ethically possible for this patient when an MRI report is available.

\section{Conclusion}

Our study did not find any significant difference between the overall prostate cancer and CSPCa detection rates between TRUS-SB and COG-TB. Therefore, considering the costs and the country's economy, standard systematic TRUS-SB may be sufficient, especially in patients who initially underwent prostate biopsy. However, we think that it would be more appropriate to perform rebiopsy under MRI guidance in patients with a previous history of a negative biopsy.

\section{Acknowledgements}

Publication: The results of the study were not published in full or in part in form of abstracts.

Contribution: There is not any contributors who may not be listed as authors.

Conflict of Interest: No conflict of interest was declared by the authors.

Financial Disclosure: The authors declared that this study received no financial support.

\section{Ethics}

Ethics Committee Approval: This study was conducted in accordance with the principles of the Helsinki Declaration and approval of the Ethics Committee of our institute was obtained (register no: 2018/15-14).

Informed Consent: Informed consent forms were obtained.

Peer-review: Externally peer-reviewed.

\section{Authorship Contributions}

Concept: O.A., M.E., M.N.B., E.D., B.K., S.Ç., S.S.Ç., A.H.T., Design: O.A., M.E., M.N.B., B.K., S.Ç., S.S.Ç., A.H.T., Data Collection or Processing: O.A., M.E., M.N.B., E.D., S.Ç., S.S.Ç., Analysis or Interpretation: O.A., M.E., M.N.B., A.H.T., Literature Search: O.A., M.E., M.N.B., E.D., B.K., S.S.Ç., Writing: O.A., M.E., E.D., S.Ç.

\section{References}

1. Bray F, Ferlay I, Soerjomataram I, et al. Global cancer statistics 2018: GLOBOCAN estimates of incidence and mortality worldwide for 36 cancers in 185 countries. CA Cancer J Clin 2018;68:394-424.
2. Hodge KK, McNeal JE, Terris MK, Stamey TA. Random systematic versus directed ultrasound guided transrectal core biopsies of the prostate. J Urol 1989;142:71-74; discussion 74-75.

3. Babaian RJ, Toi A, Kamoi K, et al. A comparative analysis of sextant and an extended 11-core multisite directed biopsy strategy. J Urol 2000;163:152-157.

4. Kam J, Yuminaga $Y$, Kim R, et al. Does magnetic resonance imagingguided biopsy improve prostate cancer detection? A comparison of systematic, cognitive fusion and ultrasound fusion prostate biopsy. Prostate Int 2018;6:88-93.

5. Jones JS, Patel A, Schoenfield L, et al. Saturation technique does not improve cancer detection as an initial prostate biopsy strategy. J Urol 2006;175:485-488.

6. Taira AV, Merrick GS, Galbreath RW, et al. Performance of transperineal template-guided mapping biopsy in detecting prostate cancer in the initial and repeat biopsy setting. Prostate Cancer Prostatic Dis 2010;13:71-77.

7. Barentsz JO, Richenberg J, Clements R, et al. ESUR prostate MR guidelines 2012. Eur Radiol 2012;22:746-757.

8. Yamada Y, Shiraishi T, Ueno A, et al. Magnetic resonance imagingguided targeted prostate biopsy: Comparison between computersoftware-based fusion versus cognitive fusion technique in biopsynaïve patients. Int J Urol 2020;27:67-71.

9. Shen WW, Cui LG, Ran WQ, et al. Targeted biopsy with reduced number of cores: optimal sampling scheme in patients undergoing magnetic resonance Imaging/transrectal ultrasound fusion prostate biopsy. Ultrasound Med Biol 2020;46:1197-1207.

10. Weinreb JC, Barentsz JO, Choyke PL, et al. PI-RADS prostate imaging reporting and data system: 2015, version 2. Eur Urol 2016;69:16-40.

11. Matoso A, Epstein JI. Defining clinically significant prostate cancer on the basis of pathological findings. Histopathology 2019;74:135-145.

12. Moore CM, Kasivisvanathan V, Eggener $S$, et al. Standards of reporting for MRI-targeted biopsy studies (START) of the prostate: recommendations from an International Working Group. Eur Urol 2013;64:544-552.

13. Bray F, Kiemeney L. Epidemiology of prostate cancer in Europe: patterns, trends and determinants. In: Bolla $M$, van Poppel $H$, eds. Management of Prostate Cancer: A Multidisciplinary Approach. Berlin, Springer-Verlag, 2017;1-11.

14. Schoots IG, Roobol MJ, Nieboer D, et al. Magnetic resonance imaging-targeted biopsy may enhance the diagnostic accuracy of signifcant prostate cancer detection compared to standard transrectal ultrasound-guided biopsy: a systematic review and metaanalysis. Eur Urol 2015;68:438-450.

15. Hu X, Yang ZQ, Shao YX, et al. MRI-targeted biopsy versus standard transrectal ultrasound-guided biopsy: a systematic review and meta-analysis of randomized controlled trials. Abdom Radiol (NY) 2020;45:3283-3292.

16. Kasivisvanathan $\mathrm{V}$, Rannikko AS, Borghi $\mathrm{M}$, et al. MRI-targeted or standard biopsy for prostate-cancer diagnosis. N Engl J Med 2018;378:1767-1777.

17. Baco E, Rud E, Eri LM, et al. A Randomized Controlled Trial To Assess and Compare the Outcomes of Two-core Prostate Biopsy Guided by Fused Magnetic Resonance and Transrectal Ultrasound Images and Traditional 12-core Systematic Biopsy. Eur Urol 2016;69:149-156.

18. Carlsson SV, Holmberg E, Moss SM, et al. No excess mortality after prostate biopsy: results from the European Randomized Study of Screening for Prostate Cancer. BJU Int 2011;107:1912-1917.

19. Anastasiadis A, Zapala L, Cordeiro E, et al. Complications of prostate biopsy. Expert Rev Anticancer Ther 2013;13:829-837.

20. Ecke TH, Gunia S, Bartel P, et al. Complications and risk factors of transrectal ultrasound guided needle biopsies of the prostate evaluated by questionnaire. Urol Oncol 2008;26:474-478. 